\section{Resolution Confusion}

\author{
Jerry Sedgewick \\ University of Minnesota \\ sedge001@umn.edu
}

The most persistent misconception made in digital imaging among those who use our core facility involves the understanding of digital resolution; and the greatest mistakes result from a mis-use of the "Image Size" dialogue box in Adobe Photoshop. Add to that, an intractable desire to use Microsoft PowerPoint and to somehow make Photoshop and PowerPoint agree about the nature of images that open in both programs, weighted toward the miserable resolutions and compression required by PowerPoint, changes in original data become as common as dandelions on a lawn. Yet, changes in data that result from increasing or, more commonly, decreasing pixels (what is called re-sampling), can be avoided.

Resolution, as many of us have learned, is the ability to discriminate between two points in space, a phenomenon made easier by high contrast objects versus those that are low in contrast. Following that definition, the caveat for digital imaging would then be a desire for 1) more pixels that are 2) physically smaller in size, and 3) spaced more closely together; since the pixel itself can be the limiting component of an image after optics and scattering of light. Thus, in the simplest sense, more pixels equals greater possibility for resolution.

Digital camera manufacturers are well aware of that, especially those who market for mass consumption. Every month we read about mega amounts of mega pixels, with the implicit understanding that more mega pixels are good. The reported resolution from many of these cameras differs, however, from the advertised resolution because of two things:

1. Only two-thirds of the pixels are typically used

2. Visual information is "grouped" using lossy compression.

Many of these cameras use the cheaper "mosaic" chip. Since the photosites (the physical photon detection sites that typically correlate $1: 1$ with subsequent pixels) on a chip simply read photons which are "colorless" (since chips only "see" in grayscale), red, green and blue microfilters are placed on each photosite. These filtered photosites are subsequently divided into what are called "channels" in Photoshop, and intensities of each channel are averaged together by taking several photosites and averaging the color in that location. In that process, fully a third of all pixels are "thrown out." Thus, it is a good idea when buying a camera to ask if a mosaic chip is used
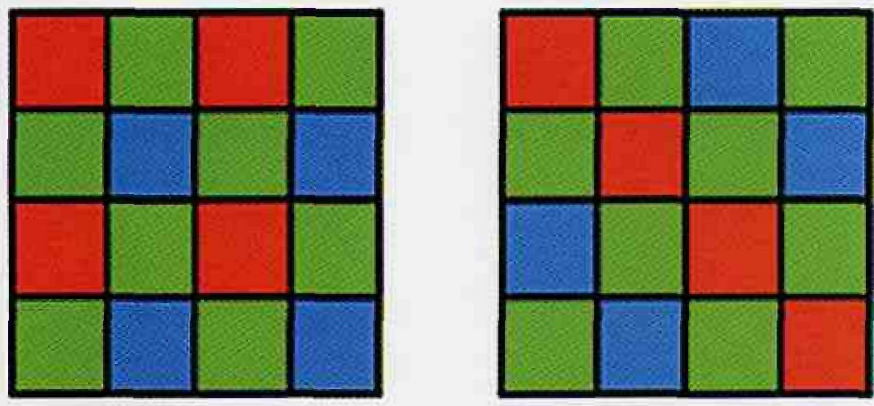

MOSAIC CHIP LAYOUTS

Note the predominant use of green

Resolution can be further reduced by compression into the commonly used JPEG format. Some JPEG compression is non-lossy - in essence no pixels are averaged together-but most JPEG compression averages color (versus intensities) even further. Camera manufacturers have found that the human is more offended by changes and alterations in contrast than in color. Thus, resolution loss along the lines of finely divided areas of color and consequent detail again takes the back seat.

Often, a camera with less pixels across and down better fits our needs. Those who work with fluorescently labeled tissue at magnifications greater than what is achieved with a $40 x$ lens need FEWER pixels than those who work at lower magnification with bright field, or those who work in electron microscopy. The upper end of magnification in light microscopy suffers a loss of resolution far more from optics and the nature of the material that is imaged than by how many pixels are used. In other areas of imaging, such as when video-rates of 30 frames per second need to be achieved and that digital information stored on a computer, less pixels across and down becomes a necessity, because of current limitations with fast imaging and the practicality of wading through immense amounts of data and massive amounts of disk space.

Whatever the beginning resolution is, and how that was maintained through a chip whose sum of advertised pixels across and down often operates as a megalie, the result is an image on the computer screen that now has a resolution that is fixed. That resolution has only one reference: the number of pixels across and down, viewed in Photoshop's Image Size dialogue box as the height and width (seen only when resample is checked in Photoshop $5 x-7 x$ ). It should be stated that the inherent resolution of an image is not necessarily what a person discriminates on a computer screen, for that is limited by the resolution of the screen itself, something that has far less resolution than a typical printer. Do not be fooled by the magnification indicator at the top, left of the image window, either: that percentage is simply the size of the image in relation to the dimensions of your computer screen display settings (thus an image that is 800 pixels in width reads at a magnification of $100 \%$ when it fills a screen in which the screen settings are also set to a width of 800 pixels).

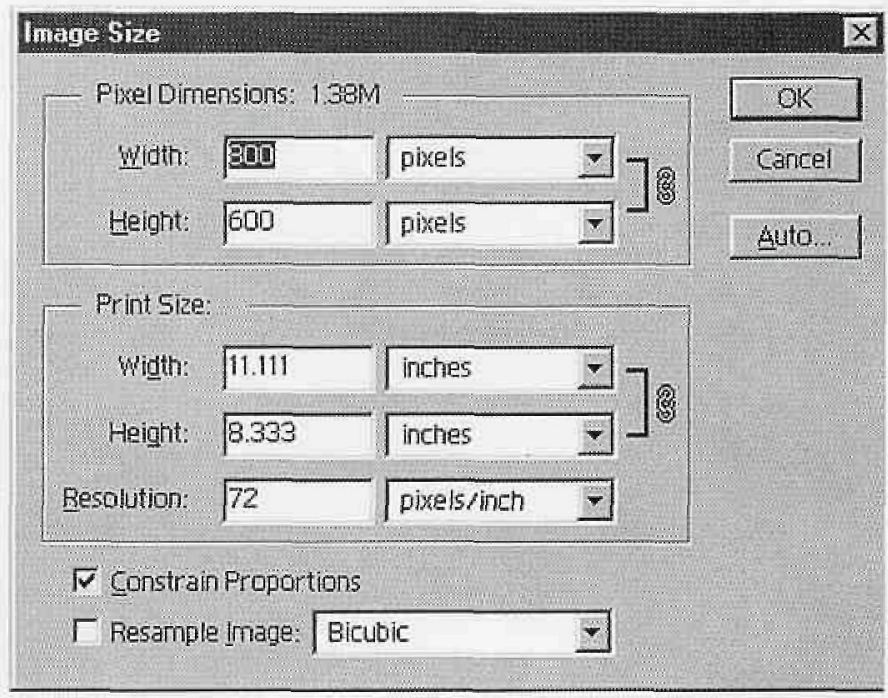

The Width and Height values at the top of the dialoue box indicate the inherent or "real" resolution of the image. The Resolution value near the bottom of the dialogue box does NOT show the real resolution, but the output resolution when the image is printed, Resample Image should remain unchecked when keeping image at original resolution.

The Image Size dialogue box is the great pitfall for many. Again, we encounter a sort of lie: in the Image Size dialogue box we read "resolution" and that is a measurement of pixels per inch. Fully aware that the inherent resolution of the image is its height and width in pixels, and then seeing another definition of resolution called "pixels per inch" immediately creates confusion. The makers of Photoshop call pixels per inch (ppi) "resolution" and so that must be true. But it isn't. The makers of Photoshop should have labeled ppi "output resolution," because pixels per inch refers to what the resolution will be when it is printed, and then only when it is printed to a printer that uses ppi as a parameter, (what is true, for example, with the Fuji Pictrography printer). When a printer uses dots per inch or lines per inch as a means for measurement, then, once again, we encounter a measurement that is untrue because pixels don't correlate $1: 1$ with dots made by laser and ink jet printers, nor do pixels correlate with the lines per inch measurement used by output devices for printing presses.

Furthermore, if images are acquired with a digital camera or a laser/ PMT system such as a confocal, rather than a flatbed scanner, the value placed by Photoshop in Resolution is arbitrary. Because you were not given the option to set output resolution from the beginning as pixels or dots per inch, as you would do when using a flatbed scanner, Photoshop has no way to figure it, and so an arbitrary value of either 72 or 96 ppi magically appears. Many have had the dictum of "72 dots per inch is too low" hammered into their consciousness, and so the immediate response is to 


\section{Discover the digitall lab!}

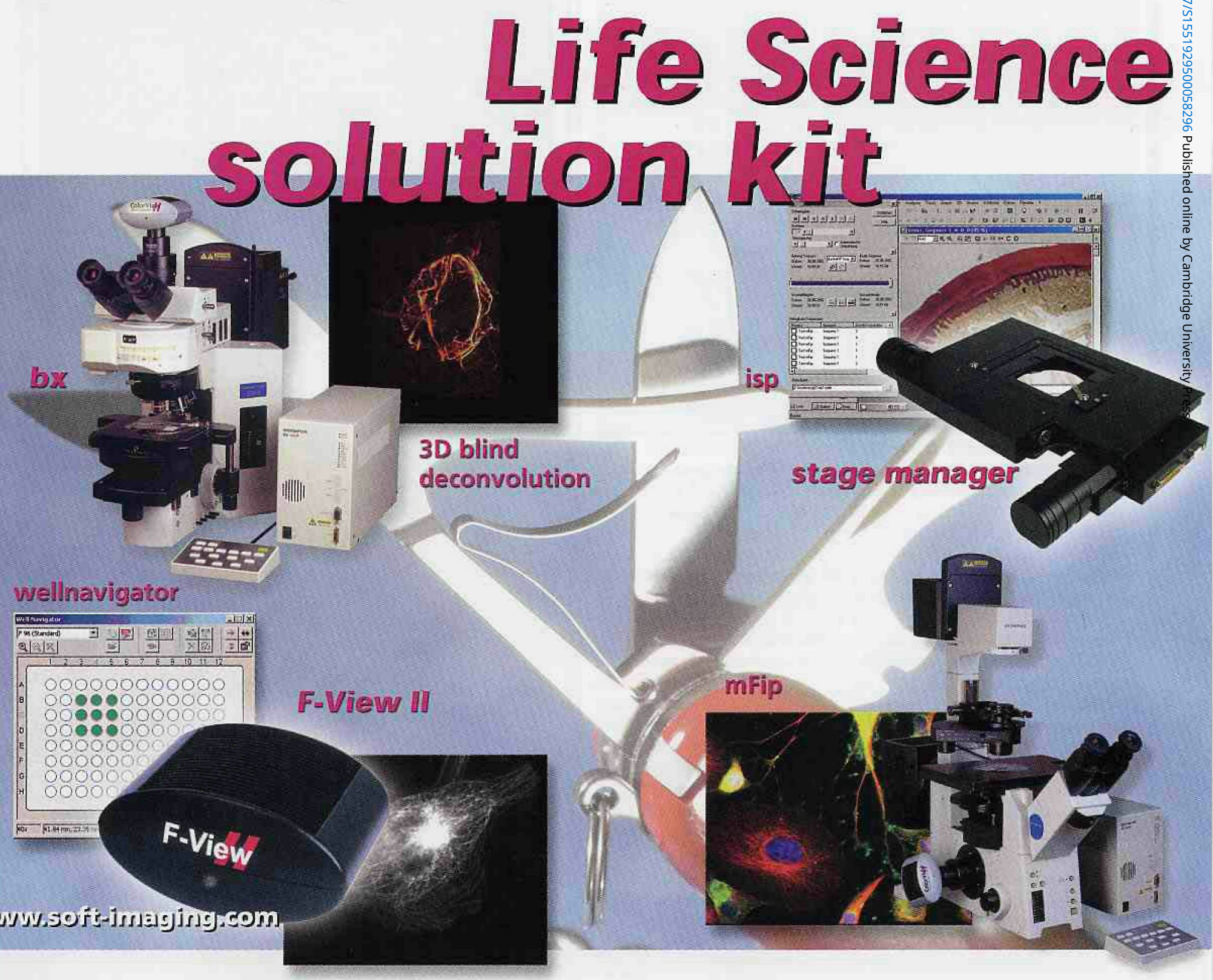

Getting integrated answers to all your questions!

Questions being dealt with in today's cell-imaging field are as varied as they are distinct. And the solutions place tough demands on software and hardware. Soft Imaging System's answer is "digital integration" with its analySIS* imageanalytical software.

analySIS ${ }^{\oplus}$ makes sure that your routine runs perfectly, provides you with easy-to-use data management including device control, as well as subsequent documentation and archiving capability.

Use analySIS ${ }^{\star}$ to successfully deal with the following areas

- controlling automated microscopes $\mid$ analySIS ${ }^{\circ}$ control - time-lapse experiments | analySIS ${ }^{\circ}$ isp + stage
- multichannel fluorescence applications | analySIS ${ }^{\circ}$ mFip a deconvolution, 3-D blind deconvolution $\mid$ analySIS ${ }^{\star}$ ride - fast image acquisition / analySIS ${ }^{\infty}$ fis

- 3-D reconstruction $\mid$ analySIS ${ }^{\oplus}$ 3D

- and automatic particle analysis | analySIS ${ }^{\circ}+$ wellnavigator.

Like to know more about analysis ${ }^{\oplus}$

Cell Imaging and Soft Imaging System...?

Please visit us at:

www.soft-imaging.com +49 (251) 798000 Asia / Pacific +60 (3) 83181400 North America (888) Find SIS +1 (303) 2349270 


\section{Canras Size}

Current Size: $1.38 \mathrm{M}$

Width: 800 pixels

Height: 600 pixels

New Size: $1.38 \mathrm{M}$

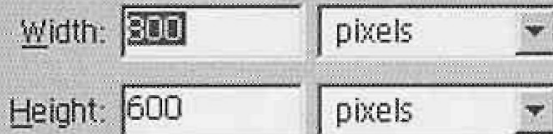

Anchor:

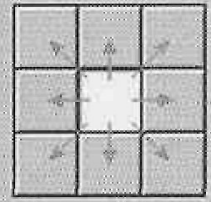

Canvas size can be used to add more space to the top, bottom, or sides of your image without changing resolution, as though you had started a painting and then needed to add more canvas space to it. This is a better way to add white space when making a multi-image figure.

raise that value to a more reasonable $300 \mathrm{ppi}$. Don't do it! You should retain original data if you wish to follow Good Laboratory Practices.

What is listed as the Height and Width in pixels in the Image Size dialogue box should not change until just before the file is sent to a printer. To be sure change doesn't accidentally happen, uncheck Resample. If two or more images need to be added to make a figure for publication or for presentation, make extra space by using the Canvas Size function under Image in the menu. You should figure what extra space is needed by adding pixels, making additional room for spaces between images. The location of the first image can be fixed by using the grid in the Canvas Size dialogue box.

Pixels should not be thrown away by consciously creating less pixels in height and width for the sake of squeezing images into PowerPoint, or because of a fear of large file sizes (hard disk space and faster computers are reasonably affordable). If a smaller image is needed for PowerPoint, keep the original as a TIFF or PSD (Photoshop) file in a folder entitled "original images" and save a duplicate image in the JPEG format (PICT format for earlier Macintosh computers) for PowerPoint and Microsoft Word.

Just before printing, open the Image Size dialogue box under Image, and then ONLY check Resample when creating a file for electronic submission to a publication at their recommended output resolution settings, or when printing to a photographic or dye sublimation printer in which pixels per inch may also need to be set. When printing to an ink jet printer, do NOT check "Resample." Instead, put in the Height and Width values and let the resolution setting change arbitrarily since the ppi is not translatable into the spitting of ink on paper in this context. Printing to a laser jet to evaluate images from Photoshop is not recommended, since these printers reinterpret the image in ways that do not show accurate results.

By following these steps, original pixel resolutions from your source can be maintained, and chunky or pixilated results can be avoided (with the exception of blowing up images captured at $<500$ pixels in one dimension to full page sizes and with the exception of blowing these images up far too great on a computer screen). Even better, when the time comes to publish, that one special image can be at the appropriate resolution to make the perfect cover photo for a journal.

About the author:

Jerry Sedgewick is the director of a light microscopy/digital imaging facility at the University of Minnesota. We are indeed fortunate that Jerry has agreed to write a regular Photoshop column for Microscopy Today.

Editor

\section{QuickPHOTOSHOP FOR RESEARCH:} A Guida to Digital langing for Phatashop $4 x, 5 x, 6 x .7$

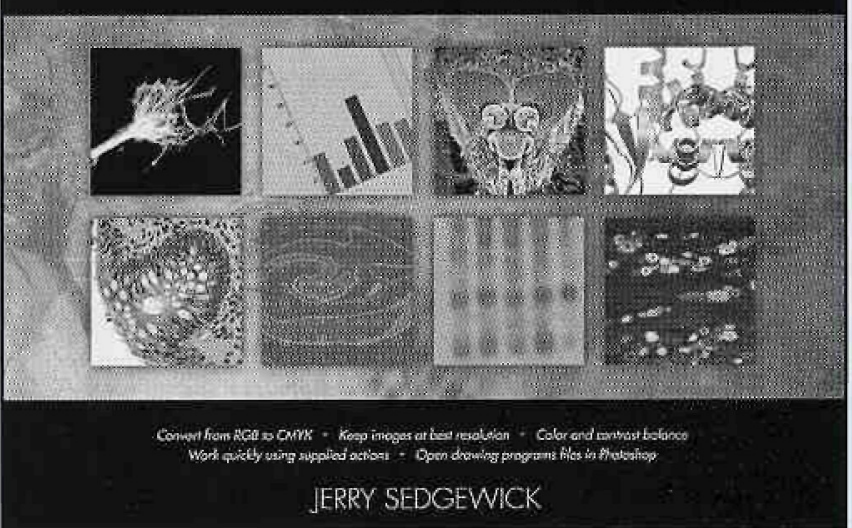

\section{Gerald Sedgewick:}

Biomedical Image Processing Lab, Dept. of Neuroscience, University of Minnesota, MN, USA

Quick Photoshop for Research: A Guide to Digital Imaging contains essential information on the use of Photoshop specific to researchers. This step-by-step spiral-bound guide is the only book published for users whose needs are not for the purpose of graphic or web design: instead, this book only addresses the tools and functions necessary for the ethical enhancement of scientific images, and subsequent layout of these images into figures or plates. The aim is to provide information about digital imaging in an easy-to-follow guide from the beginning of the imaging process to its end.Additional information about scanning and acquiring images via a digital camera or laser/PMT system is also covered, as well as information about printers and PowerPoint.

\section{Contents:}

Part I: Before Getting Started in Photoshop. Flatbed Scanners. Film Scanners. Scanning Tips. Digital Camera Systems. Making Hon-pizelated, High Resolution Images from Graphing, Drawing \& Word Processing Programs. Differences Between a Drawing Program (Vector Graphics) and a Paint Program (Image Files). Ethics and Phatoshop. Computer Requirements for Running Photoshop. Computer Screens (monitors). Setting Memory for Photoshop on a Macinnosh. Visual Definitions. Overview of Toolbar. Setring up The Photoshop Workspace. Troubleshooting. Load Actions in Photoshop, Part 2: Quick Photoshop: Steps for Single Images. Marquee Tool and Guidelines. Open Image/Duplicate. Rotate/flip. Undo \& History. Zooning In \& Out. Crop. Mode. Dust and Scratches. Sharpening. Contrast \& Color: A Primer. Contrasu/Color Auto Actions. Contrasu/Color Manual Adjustments. ContrastColor Darkfield \& Drawings or Graphs. Contrast: Measuring Pixels \& Matching Background Gray Levels. Manual Color Controls/Subtle colors.Color-Changing Certain Colors. Adding Color, Subsequent Steps for Single Image. Final Resolution and Dimensions for Single Images.

Part 3: Combining More Than One Image and Adding Lettering. Layers. Layers: Actions \& Flattening. Cropping Several Images to the Same Dimensions. Merging Images. Combining $>1$ Image. Color-Changing Certain Colors. Adding Color. Adding White Space. For Lettering in Photoshop. Manual Lettering of Images $(4 x, 5 x)$ Lettering in Photoshop $(6 x, 7 x)$ Enhancements to Lettering Alignment and Orientation of Type

Part 4: Finalizing Figures. Auto, Single \& Multi-Image Figures (or Plates). Manual Layout of Multiple Images to Make a Figure or Plate. Symbols. Adding Symbols Manually. Filling Image Areas with Patterns. Magnification and Scale Bars. Saving Files. Prints and $35 \mathrm{~mm}$ Slides From Digital Files. Setting Resolution and Dimensions for Output. Working With Color CMYK Files (for Publication and Pre-Press Printers). Working with PowerPoint. Color Shifts with Grayscale Photographs. Organizing \& Archiving Files Sumimary of Steps. Index.

\section{kluwer}

\section{the language of science} 2002, ISBN 0-306-47375-5, Spiral-bound paperback, 107 pP. $\$ 49.50 / E 52.50 / € 35.00$

Order online at www.wkap.com 


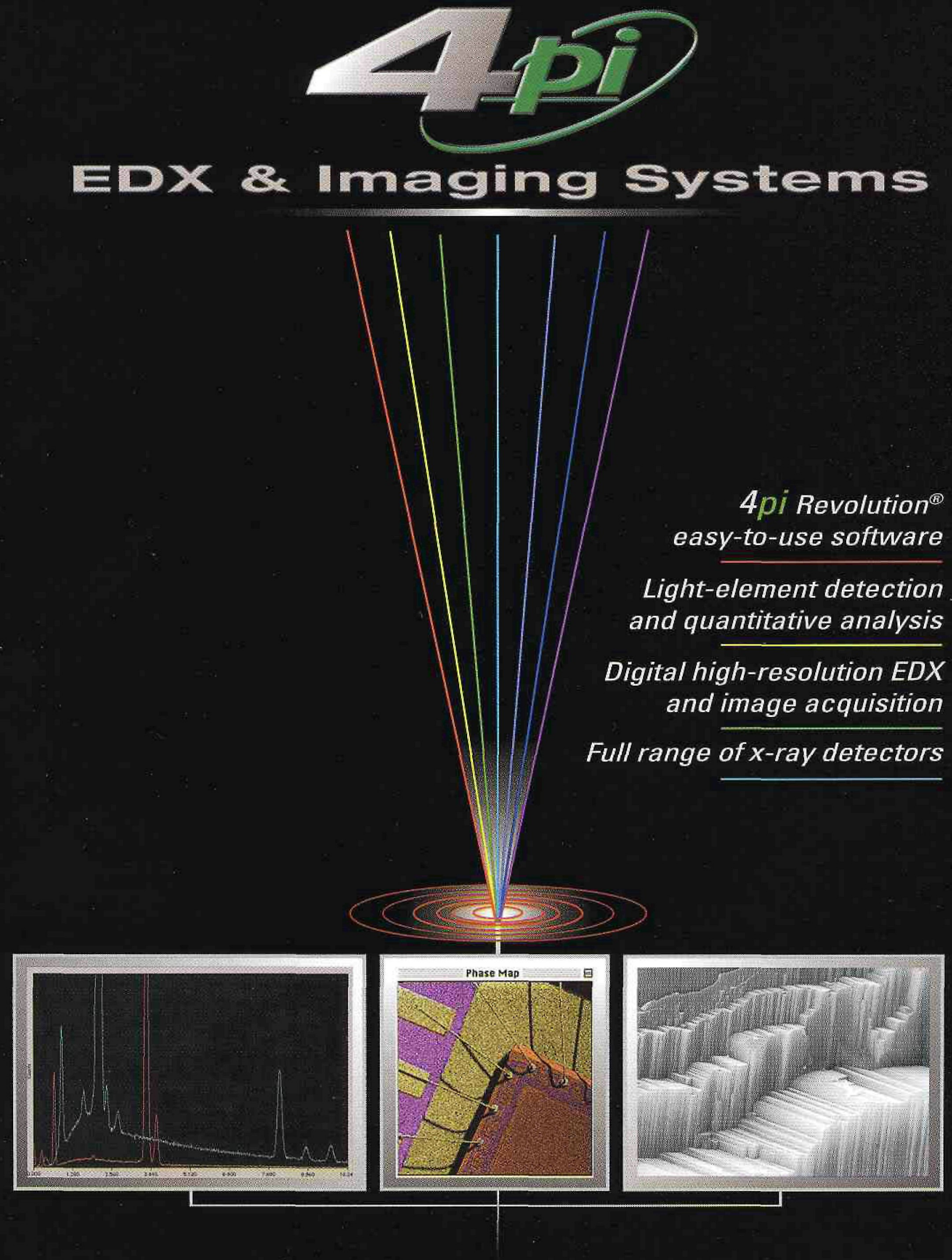

